\title{
Impact of Postoperative Radiotherapy and Chemotherapy on Survival for Patients with Node Positive Oral Cancer
}

\author{
Mohamed Mahmoud ${ }^{1}$, Tarek Shouman ${ }^{1}$, Hesham Elhosseny ${ }^{1}$, Mona Aboelenin ${ }^{2}$, Mohamed Amin ${ }^{1}$ \\ ${ }^{1}$ Radiation Oncology Department, National Cancer Institute, Cairo University, Cairo, Egypt; ${ }^{2}$ Clinical Oncology Department, Faculty \\ of Medicine, Cairo University, Cairo, Egypt. \\ Email: m_mahmoud1973@yahoo.com
}

Received August $25^{\text {th }}, 2013$; revised September $22^{\text {nd }}, 2013$; accepted September $30^{\text {th }}, 2013$

Copyright (C) 2013 Mohamed Mahmoud et al. This is an open access article distributed under the Creative Commons Attribution License, which permits unrestricted use, distribution, and reproduction in any medium, provided the original work is properly cited.

\begin{abstract}
Purpose: The aim of the present study is to compare between the use of post-operative concurrent chemotherapy and radiotherapy alone on survival for patients with high risk oral cavity tumors at the National cancer Institute of Egypt, undergoing surgery and receiving adjuvant treatment. Patients and Methods: This is a retrospective study, which was carried out at the National Cancer Institute (Cairo University) on patients with node positive oral cavity cancer diagnosed between the year 2000 and 2008. The study included 60 patients ( 45 males and 15 females) with median age 57 years old. The patients underwent surgery, followed by postoperative radiotherapy $60 \mathrm{~Gy} / 6$ weeks versus postoperative radiotherapy $60 \mathrm{~Gy} / 6$ weeks with concurrent cisplatin $100 \mathrm{mg} / \mathrm{m}^{2}$ at day 1, day 22 and day 43. Results: Regarding use of concurrent chemotherapy, there was a significant difference in overall survival rate and locoregional control favoring patients who received concurrent chemotherapy and radiotherapy, 3 years and 5 years overall survival rates respectively were $53.8 \%$ and $40.4 \%$ compared to $37.5 \%$ and $26.3 \%$ for patients who didn't receive any chemotherapy with (p < 0.038 ) for 5 years. Regarding age, there was a significant difference in overall survival rate favoring patients $\leq 57$ years in both arm groups, 3 years and 5 years overall survival rates respectively were $51.6 \%$ and $38.9 \%$ compared to $28.3 \%$ and $18.9 \%$ for patients $>57$ years with $(\mathrm{p}<0.028)$ for 5 years. Conclusion: We recommend for oral cavity tumor patients at the NCI of Egypt who have positive neck nodes to be treated with concurrent chemo-radiotherapy rather than radiotherapy alone especially by using the new techniques as intensity modulated radiotherapy (IMRT) and image guided radiotherapy (IGRT).
\end{abstract}

Keywords: Concurrent Radiotherapy and Chemotherapy; Intensity Modulated Radiotherapy; Image Guided Radiotherapy

\section{Introduction}

It is generally accepted that oral cavity squamous cell carcinoma (OSCC) patients with positive lymph nodes, Stage pT4 disease, or positive/close surgical margins require adjuvant radiotherapy (RT).

The application of chemotherapy to the treatment of head and neck cancer dates back to the 1960s. Over the decades, the role of chemotherapy has advanced from initial use only in the recurrent or metastatic setting to active current use in the definitive treatment setting. Cooper et al. 2004 [1] reported the results of a randomized study in North America comparing radiation alone (60 to $66 \mathrm{~Gy}$ ) to chemoradiation (same radiation dose plus three cycles of $100 \mathrm{mg} / \mathrm{m}^{2}$ cisplatin) in patients with head and neck carcinoma demonstrating high-risk features after gross total resection.

This study demonstrated a benefit in loco-regional control and disease-free survival for the chemoradiation arm, but no overall survival benefit was appreciated. Bernier et al. 2005 [2] randomized patients to essentially equivalent treatment arms following head and neck cancer surgery. Eligibility criteria included patients with pathologic T3 or T4 disease (except T3/N0), or patients with any T-stage disease with two or more involved lymph nodes, or patients with T1-2 and N0-1 disease with unfavorable pathologic findings (extranodal spread, positive margins, perineural involvement, or vascular embolism). Local control, progression-free survival, and overall survival were superior for patients on the chemoradiation 
arm. These studies suggest that the addition of chemoradiation following surgery may be beneficial in selected patients with high-risk head and neck cancer, although with increased toxicity profiles.

The aim of the present study is to detect whether the use of post-operative concurrent chemotherapy and radiotherapy has an impact on survival than post-operative radiotherapy alone on patients who underwent surgery and received adjuvant treatment, with high risk oral cavity tumors treatment at the National Cancer Institute of Egypt.

\section{Patients and Methods}

This is a retrospective study, which includes 60 patients with node positive squamous cell carcinoma of the oral cavity. The patients underwent surgery, followed by postoperative radiotherapy or postoperative radio-chemotherapy at the radiotherapy department, National Cancer Institute, Cairo University during the period between 2000 and 2008.

Data obtained from the patients' files included, patients serial number, age, sex, history of smoking, date of biopsy, date of surgery, type of neck dissection, tumor site, size, histological type, grade, surgical margin, lymph node state, TNM, dose of chemotherapy, all data of radiotherapy, time of occurrence of relapse whether locoregional or distant as well as date of the patients in last visit.

All the patients were planned through 2-D simulator planning using isocentric technique; thirty-seven patients $(33 \%)$ received unilateral treatment while seventy-five patients $(67 \%)$ received bilateral treatment.

The treated volume was either irradiated by two opposing fields in which its lower border was matched with the upper border of a separate anterior direct field to cover the neck nodes or by 2 laterals opposed fields which included the neck nodes. All patients received a dose of $60 \mathrm{~Gy} / 6$ weeks.

As regarding chemotherapy, 30 patients received Concurrent Chemotherapy after checking of $\mathrm{CBC}$, liver \& kidney functions, serum calcium \& magnesium together with proper hydration Patients received 3 cycles of chemotherapy $\left(100 \mathrm{mg} / \mathrm{m}^{2}\right.$ on D1, D22, D43).

The median follow up was for 3 years. In each visit complete physical examination was performed and investigations (in the form of $\mathrm{CBC}$, liver functions, kidney functions, local $\mathrm{CT}$ and pan endoscopy) were done every 6 months, some other investigations (chest X-ray, abdominal ultrasound and bone scan) were selectively ordered on suspicion.

Any abnormal finding was well documented and relapse was confirmed radiologically (e.g. lung, liver, bone, brain secondaries), and/or histopathologically (e.g. locoregional relapse or soft tissue secondaries).

\section{Statistical Analysis}

- Quantitative data were statistically described in terms of mean standard deviation and range, while discrete data were described using frequencies (number of cases) and percentages when appropriate.

- Comparison of numerical variables between the study groups was done using Student $t$ test for independent samples in comparing 2 groups when normally distributed and Mann Whitney $U$ test for independent samples when not normally distributed.

- For comparing categorical data, Chi square test was performed. Exact test was used instead when the expected frequency was less than 5 .

- Kaplan-Meier method was used to estimate overall survival as a function of time since start of treatment.

- Comparisons between survival functions were performed by using the log rank statistic.

- A probability value ( $\mathrm{p}$ value) less than 0.05 is considered statistically significant. All statistical calculations were done using SPSS (Statistical Package for the Social Science; SPSS Inc., Chicago, IL, USA) version 17 for Microsoft Windows.

- Overall survival was calculated from the time of diagnosis to the time of death or last follow-up.

\section{Results}

This is a retrospective study was carried out at the National Cancer Institute (Cairo University) on patients with node positive oral cavity cancer diagnosed between the year 2000 and 2008. The study included 60 patients. The patients underwent surgery, followed by postoperative radiotherapy or postoperative radio-chemotherapy.

The median patients age at diagnosis was 57.1 years (range 29 - 78 years), 30 patients received post-operative radiotherapy alone by a dose $60 \mathrm{~Gy} / 30$ fractions over 6 weeks, $2 \mathrm{~Gy} /$ fraction and 30 patients received the same schedule of radiation with concurrent cisplatin $100 \mathrm{mg} / \mathrm{m}^{2}$ at D1, D22 and D43.

The follow up period ranged between 2 - 92 months with a median follow up of 28 months. As shown in Figure 1 the overall survival rate at 3 years was $43 \%, 5$ year rate was $32 \%$ and median overall survival was 29 months (95\% CI 23.3 - 34.6).

\subsection{Univariate Analysis}

The 3 years overall survival and 5 years overall survival was calculated for all patients in both arm groups and shown in Table 1.

Regarding Nodal stage as shown in Figure 2, for patients with $\mathrm{N} 1$ disease the 3 -year overall survival rate was $40 \%$. Whereas for N2 disease was $22.2 \%$ while the 5 -year overall survival rate for patients with $\mathrm{N} 1$ disease was 


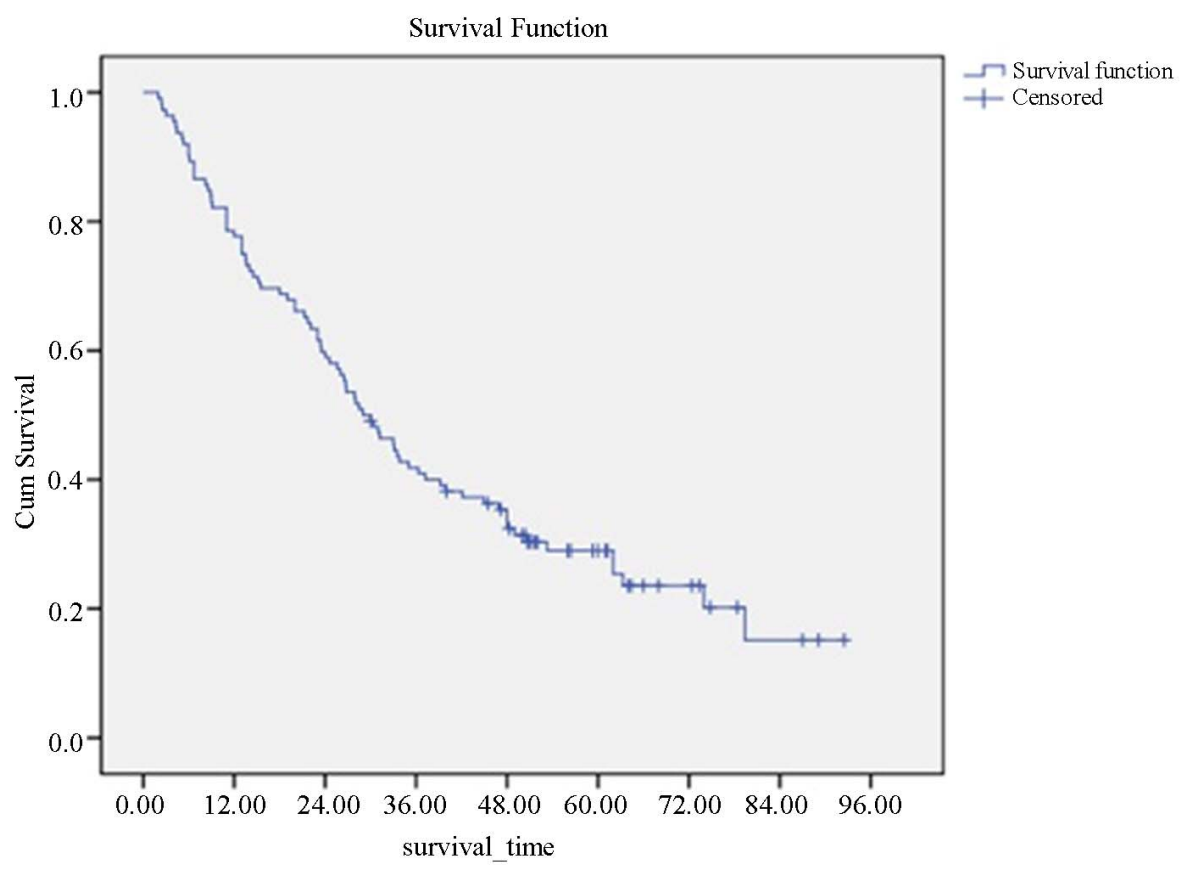

Figure 1. Overall survival for all patients.

$36.6 \%$ and for patients with $\mathrm{N} 2$ disease survival rate was $13.9 \%$ with $(\mathrm{p}<0.006)$ for 5 years.

Regarding the stage of the disease there was a significant difference in overall survival rate favoring patients with stage III; 3 years overall survival and 5 years survival rates respectively were $51.8 \%$ and $41.3 \%$ compared to $25.5 \%$ and $15.4 \%$ for patients with stage IV $(p<0.007)$ for 5 years as shown in Figure 3.

In Figure 4, regarding use of concurrent chemotherapy, there was a significant difference in overall survival rate favoring patients who received concurrent chemotherapy, 3 years and 5 years overall survival rates respectively were $53.8 \%$ and $40.4 \%$ compared to $37.5 \%$ and $26.3 \%$ for patients who didn't receive any chemotherapy with $(\mathrm{p}<0.038)$ for 5 years.

Regarding age as shown in Figure 5, there was a significant difference in overall survival rate favoring patients $\leq 57$ years, 3 years and 5 years overall survival rates respectively were $51.6 \%$ and $38.9 \%$ compared to $28.3 \%$ and $18.9 \%$ for patients $>57$ years with $(p<0.028)$ for 5 years.

Regarding surgical margin as shown in Figure 6, there wasn't a significant difference in overall survival. For patients with negative margin 3 years and 5 years overall survival rates respectively were $45.6 \%$ and $29.4 \%$ compared to $33.8 \%$ and $29 \%$ for patients with positive or closer margin with $(\mathrm{p}<0.623)$ for 5 years.

The recurrence free survival rate at 3 years was $40.1 \%$ and at 5 years was $27.3 \%$ and Median recurrence free survival was 26 months as shown in Figure 7.
In Figure 8, regarding Nodal stage, for patients with $\mathrm{N} 1$ disease the 3-year recurrence free survival was 48.6 $\%$.where as for N2 disease was $22.2 \%$ while the 5-year recurrence free survival for patients with N1 disease was $33.6 \%$ and for patients with $\mathrm{N} 2$ disease survival was $13.9 \%$ with $(\mathrm{p}<0.013)$ for the 5 years.

Regarding use of concurrent chemotherapy as shown in Figure 9, there was a significant difference in recurrence free survival rate favoring patients who received concurrent chemotherapy, 3 years and 5 years recurrence free survival respectively were $53.8 \%$ and $40.4 \%$ compared to $36.4 \%$ and $24 \%$ for patients who didn't receive any chemotherapy, with median survival 39.9 and 23.4 months respectively with $(\mathrm{p}<0.026)$ for the 5 years.

\subsection{Multivariate Analysis}

- Multivariate analysis was done for different prognostic factors affecting Overall Survival.

It was shown that the following is independent prognostic factor:

1) N-Stage of the tumor: survival for patients with N1 tumors was

Better than those of $\mathrm{N} 2(\mathrm{p}<0.005)$.

- Multivariate analysis was done for different prognostic factors affecting Recurrence free Survival.

It was shown that the following are independent prognostic factors:

1) Recurrence free Survival for patients with N1 tumors was better than those of N2 $(p<0.05)$. 
Table 1. 3 years overall survival and 5 years overall survival.

\begin{tabular}{|c|c|c|c|c|c|c|}
\hline \multicolumn{7}{|c|}{ Overall survival rate } \\
\hline Factors & Number of cases & 3 years $(\%)$ & 5 years $(\%)$ & Median (months) & P-value $^{*}$ & $95 \% \mathrm{CI}$ \\
\hline All & 60 & 43 & 32 & 29 & & $23.3-34.6$ \\
\hline \multicolumn{7}{|c|}{ Age } \\
\hline$\leq 57 \mathrm{yrs}$ & 40 & 51.6 & 38.9 & 39 & & $17.5-42.2$ \\
\hline$>57 \mathrm{yrs}$ & 20 & 28.3 & 18.9 & 25 & 0.028 & $18.5-32.6$ \\
\hline \multicolumn{7}{|c|}{ Sex } \\
\hline Male & 45 & 42.6 & 28.1 & 23 & & $24.6-41.3$ \\
\hline Female & 15 & 38.4 & 30.2 & 26 & 0.820 & $19.2-34.3$ \\
\hline \multicolumn{7}{|c|}{ PS } \\
\hline 1 & 41 & 40.4 & 30.1 & 29 & & $23.1-34.2$ \\
\hline 2 & 19 & 39.8 & 21.9 & 26 & 0.972 & $14.2-34.6$ \\
\hline \multicolumn{7}{|c|}{ Smoking } \\
\hline No & 47 & 41.6 & 33.7 & 28 & & $19.6-36.2$ \\
\hline Yes & 13 & 40 & 26.1 & 30 & 0.69 & $22.8-32.2$ \\
\hline \multicolumn{7}{|c|}{ Site } \\
\hline Tongue & 45 & 36.9 & 25.9 & 26 & & $17.2-36.1$ \\
\hline Other Sites & 15 & 45.3 & 23.4 & 33 & 0.139 & $21.2-34.3$ \\
\hline \multicolumn{7}{|c|}{ Overall survival rate } \\
\hline Factors & Number of cases & 3 years $(\%)$ & 5 years $(\%)$ & Median (months) & P-value ${ }^{*}$ & $95 \% \mathrm{CI}$ \\
\hline \multicolumn{7}{|c|}{ Grade } \\
\hline 1 and 2 & 48 & 43 & 29.9 & 30 & & $24.5-36.2$ \\
\hline 2 & 12 & 35.2 & 23.5 & 23 & 0.792 & $12.6-34.1$ \\
\hline \multicolumn{7}{|c|}{ Margin } \\
\hline Negative & 39 & 45.6 & 29.4 & 33 & & $23.6-42.4$ \\
\hline Positive/close & 21 & 33.8 & 29.0 & 23 & 0.623 & $14.1-32.6$ \\
\hline \multicolumn{7}{|c|}{ T-stage (tumor size) } \\
\hline $\mathrm{T} 1 \& 2$ & 35 & 40 & 25 & 33.5 & & $19.9-47$ \\
\hline $\mathrm{T} 3 \& 4$ & 25 & 41.1 & 30 & 28.5 & 0.89 & $22.3-44.6$ \\
\hline \multicolumn{7}{|c|}{$\mathrm{N}$-stage } \\
\hline N1 & 45 & 51.2 & 40 & 36.6 & & $24.7-47.8$ \\
\hline $\mathrm{N} 2$ & 15 & 22.2 & 13.9 & 23.5 & 0.006 & $19.6-27.3$ \\
\hline \multicolumn{7}{|c|}{ Stage } \\
\hline 3 & 31 & 51.8 & 41.3 & 39 & & $25.4-54.0$ \\
\hline 4 & 29 & 25.5 & 15.4 & 24 & 0.007 & $21.1-28.2$ \\
\hline \multicolumn{7}{|c|}{ Type of neck dissection } \\
\hline RND & 25 & 35.4 & 26.1 & 28 & & $20.1-39.2$ \\
\hline MRND & 20 & 48.4 & 38.6 & 33 & & $11.9-54.3$ \\
\hline SND & 15 & 41.1 & 23.6 & 30 & 0.635 & $17.8-42.2$ \\
\hline \multicolumn{7}{|c|}{ Chemotherapy } \\
\hline NO & 30 & 37.5 & 26.3 & 26 & & $19.8-35.5$ \\
\hline Yes & 30 & 53.8 & 40.4 & 39 & 0.038 & $12.9-65.1$ \\
\hline
\end{tabular}



for Patients with Node Positive Oral Cancer

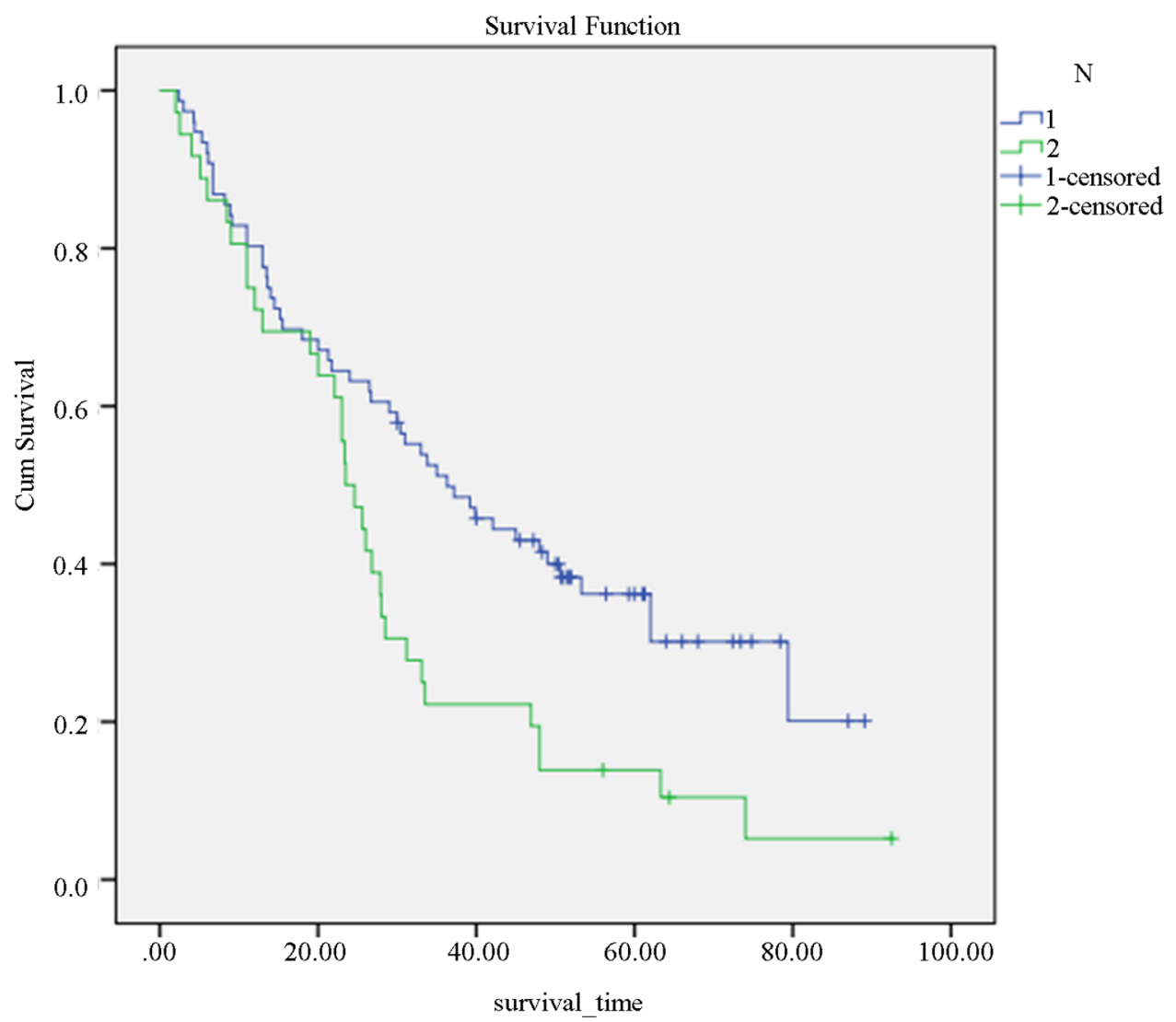

Figure 2. Overall survival according to nodal stage.

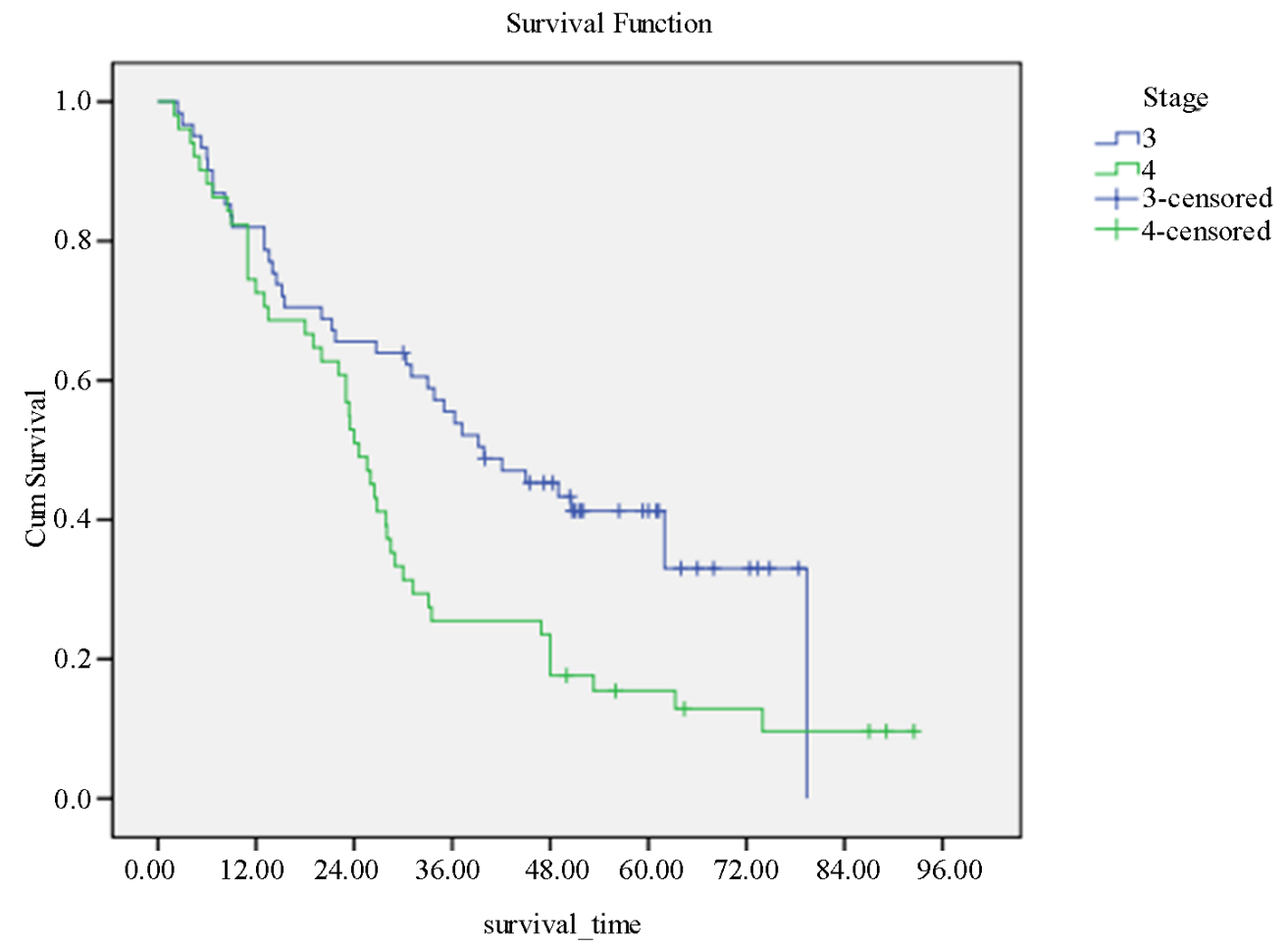

Figure 3. Overall survival according to tumor stage. 


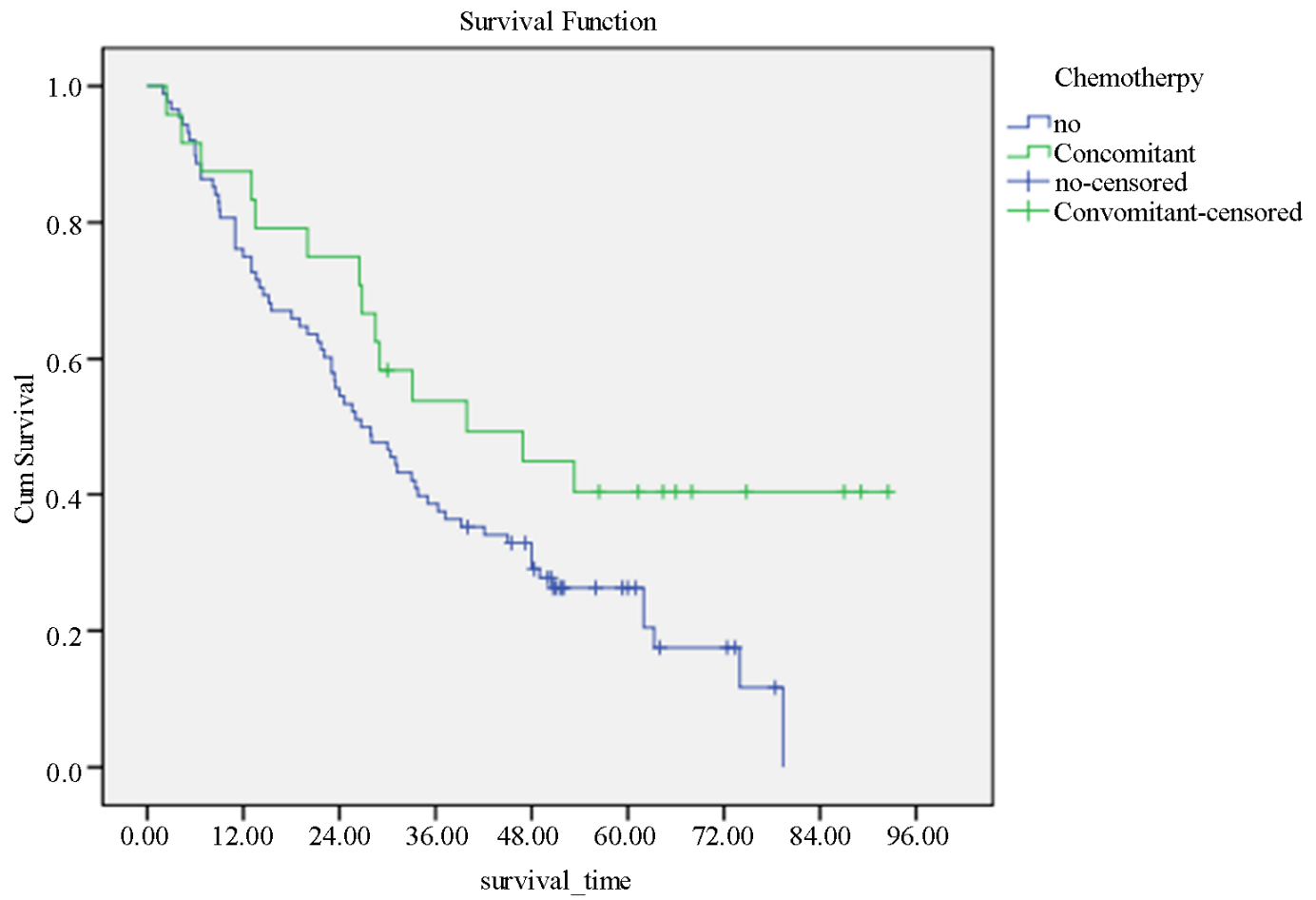

Figure 4. Overall survival according to concurrent chemotherapy.

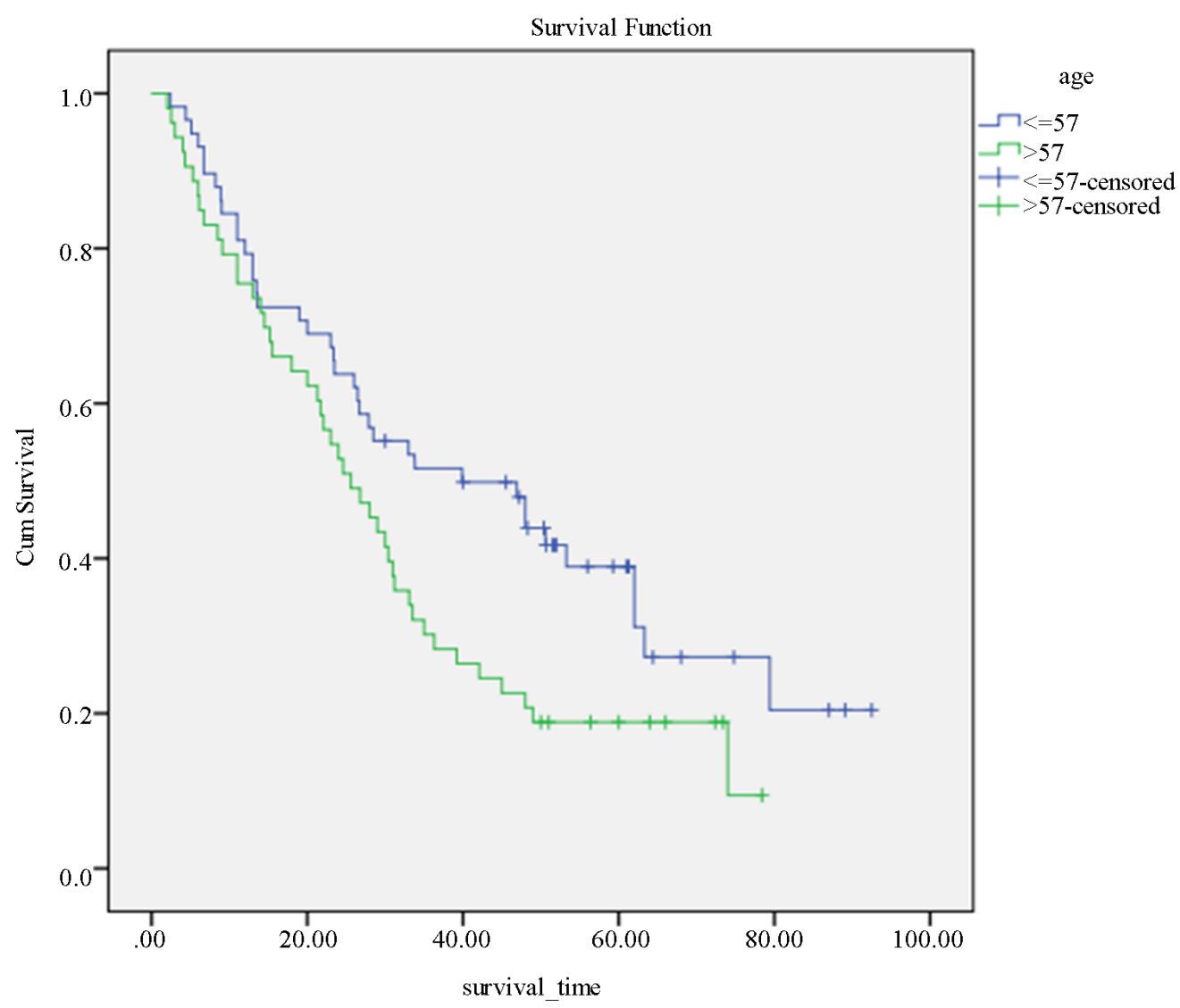

Figure 5. Overall survival according to age. 

for Patients with Node Positive Oral Cancer

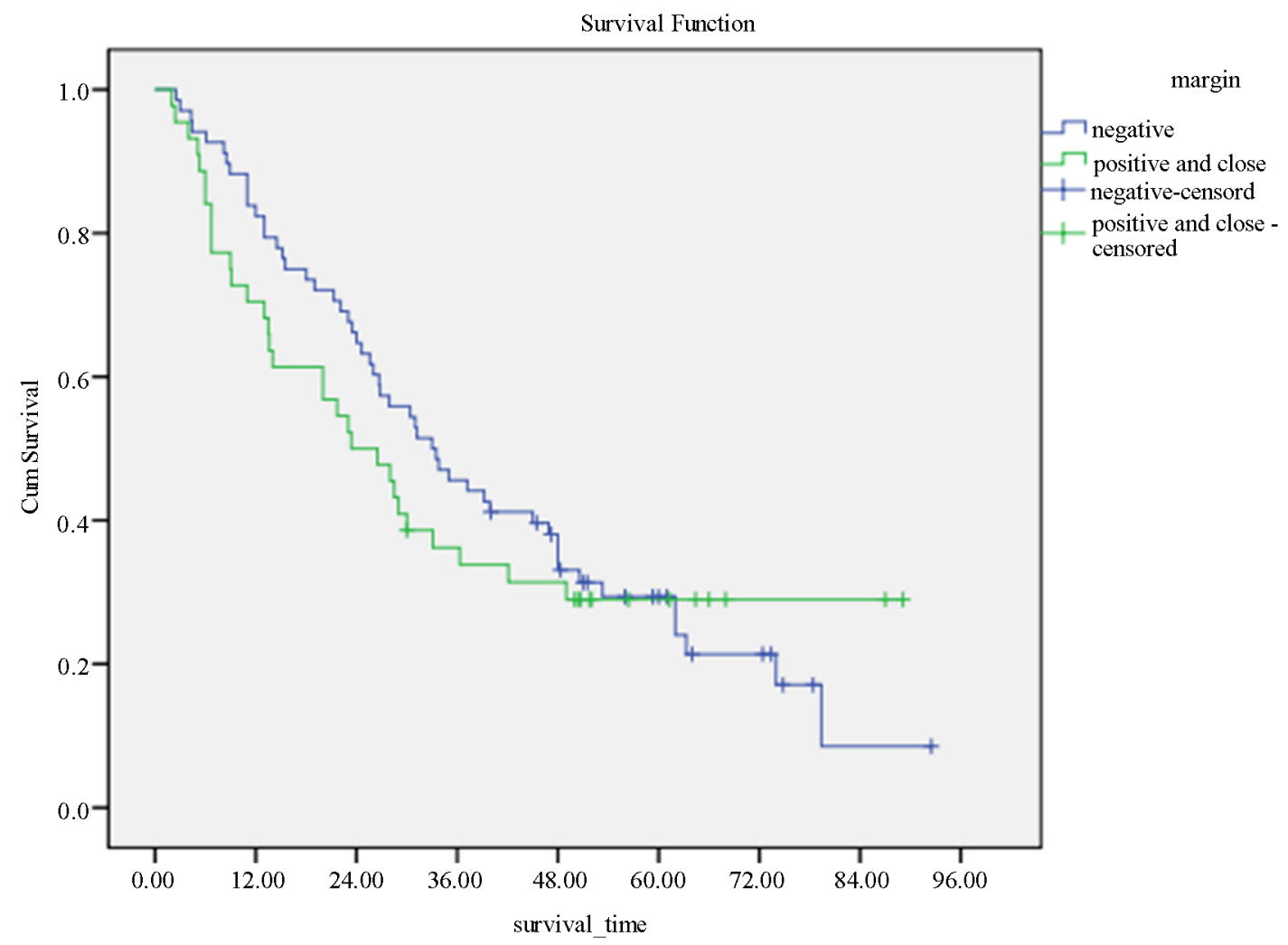

Figure 6. Overall survival according to surgical margin.

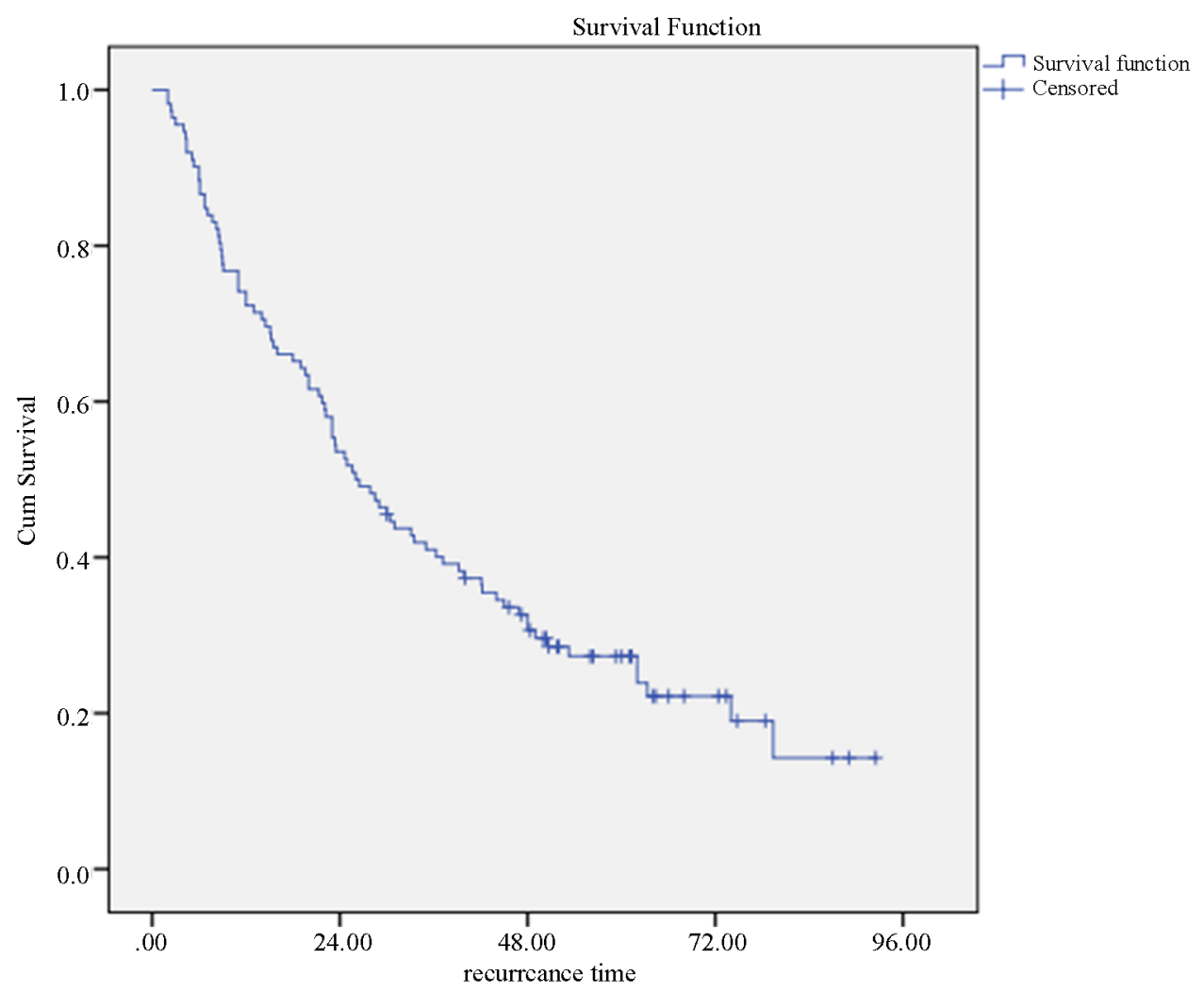

Figure 7. Locoregional control to all patients in months. 


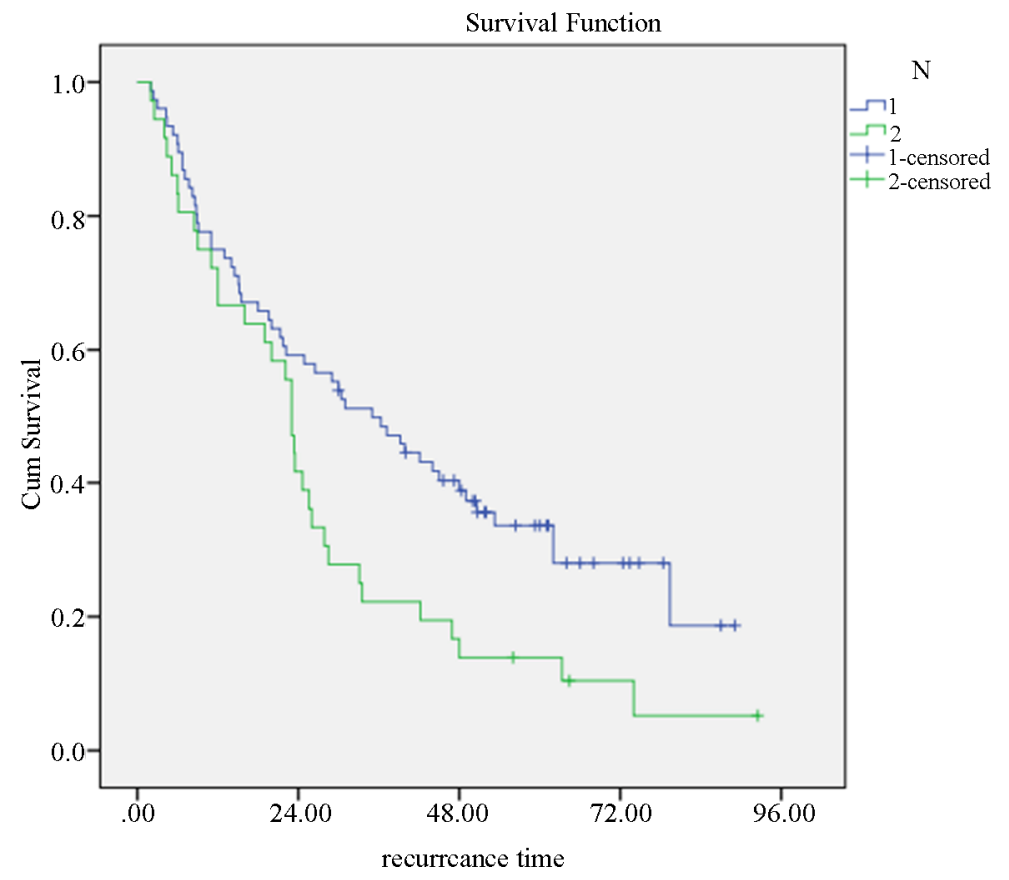

Figure 8. Recurrence free survival in months according to nodal stage.

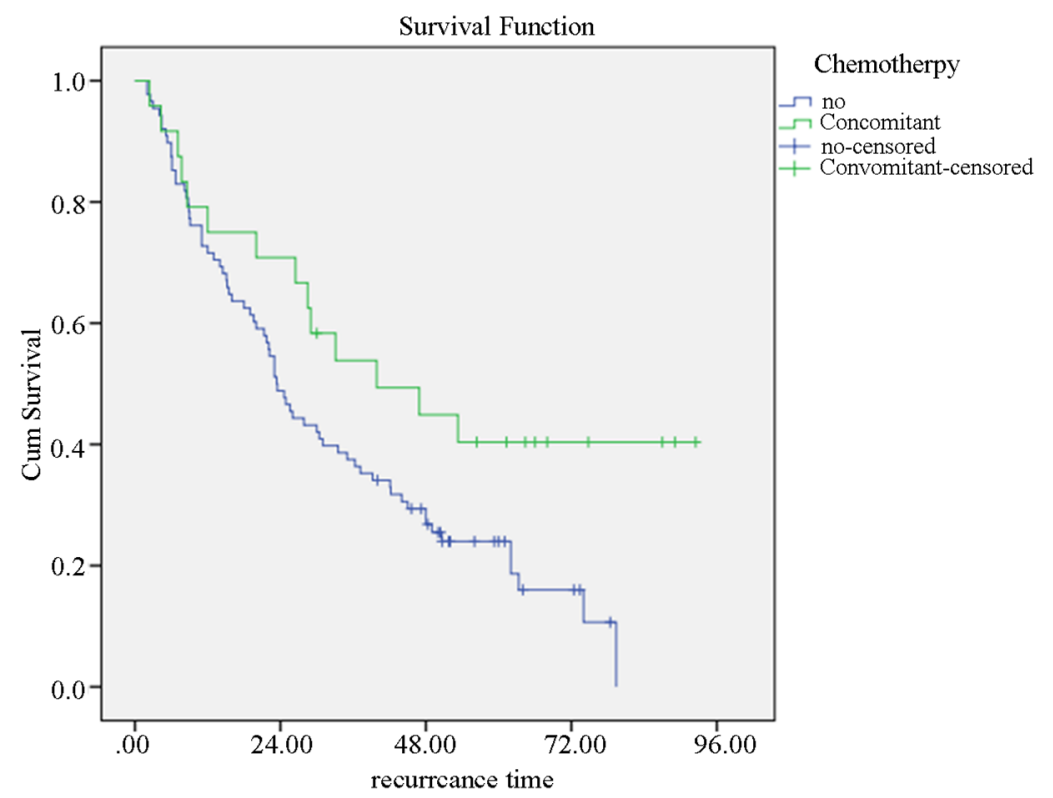

Figure 9. Recurrence free survival in months according to concurrent chemotherapy.

\subsection{Discussion}

Oral cavity tumors remain a challenging issue for oncologists despite the great advances in the multimodality approach; surgery, chemotherapy and radiation. Adjuvant radiotherapy could contribute to improved survival by improving local control of the dissected neck, primary site, pathways of perineural spread, or the undissected contralateral neck and retropharyngeal nodes.
Regarding overall survival, in our study the 3 years \& 5 years overall survival rates were $43 \%$ \& $32 \%$ respectively. Confirmatory results were reported by Bernier et al. [3] who reported that overall survival rates of patients with lymph node positive oral cavity tumors treated with surgery and adjuvant RT at 3 years and 5 years were $42 \%$ $\& 31.5 \%$ respectively. In another series [4] reported that the 3 year and 5 years overall survivals rates were $45 \%$ and $33.9 \%$ respectively. 
In our study there was significant correlation between nodal stage and overall survival (OAS), the 5-year overall survival for patients with $\mathrm{N} 1$ disease was $36.6 \%$ and for patients with $\mathrm{N} 2$ disease survival was $13.9 \%$. (p < 0.006 ).

Kao et al., 2008 [4] also reported that survival rates were affected by nodal stage and reported that N1, 2, 3 five years survival was $50.7 \%, 33.2 \%, 31.5 \%$ respectively.

In our study there was a significant correlation between concurrent chemotherapy and survival rates, 5 years overall survival for chemo-radiotherapy group was $40.4 \%$, while 5 years overall survival rates for radiotherapy alone was $26.3 \%(\mathrm{P}<0.038)$. Bernier et al., 2004 [2] reported that 5 years OAS for chemo-radiotherapy group was $56 \%$ while for radiotherapy alone $40 \%(\mathrm{P}<0.01)$.

In our study surgical margin status didn't affect OAS and local control rates, which is comparable to that reported by Hinerman et al., 2004 [5] who showed no significant correlation between surgical margin and overall survival rates $(\mathrm{p}<0.88)$.

Sandro et al. 2004 [6] treated 47 patients by postoperative cisplatin and carboplatin concomitant with radiotherapy $60 \mathrm{~Gy}$, the estimated 2-year locoregional control, progression-free survival, and overall survival rate was $73 \%, 56 \%$, and $62 \%$, respectively.

Another study conducted by Fietkau et al. 2006 [7], comparing PORT alone $66 \mathrm{~Gy} / 6.6$ weeks with the same regimen and concurrent cisplatin and 5-FU (5-Flurouracil). The 5 years LCR was $72 \%$, for PORT and $88.6 \%$ for concurrent chemoradiotherapy $(p=0.002)$, with improvement in OAS $48 \%$ for PORT versus $58 \%$ for combined modality treatment.

Branko et al. 2007 [8] subjected 114 pateints with stage III and IV SCHNC were randomized after surgery to postoperative radiotherapy alone or concomitant chemoradiotherapy. Patients in both groups were postoperatively irradiated to the total dose of $56-70 \mathrm{~Gy}$. Chemotherapy included Mitomycin C $15 \mathrm{mg} / \mathrm{rn} 2$ after 10 Gy and $5 \mathrm{mg}$ of Bleomvcin twice weekly during irradiation. Median follow-up was 76 months (48 - 103 months). At 5 years in the RT and CRT arms, the locoregional control was $65 \%$ and $88 \%(p=0.026)$, Disease-free survival $33 \%$ and $53 \%(\mathrm{p}=0.035)$, and overall survival $37 \%$ and $55 \%(\mathrm{p}=0.091)$ respectively.

\section{Conclusions}

In summary, we have demonstrated better overall survival rates in oral cavity tumor patients having positive neck nodes when treated with adjuvant concurrent chemotherapy and radiotherapy rather than adjuvant radiotherapy alone.

So we recommend for oral cavity tumor patients at the NCI of Egypt who have positive neck nodes to be treated with concurrent chemo-radiotherapy rather than radiotherapy alone specially by using the new techniques as intensity modulated radiotherapy (IMRT) and image guided radiotherapy (IGRT).

\section{REFERENCES}

[1] J. S. Cooper, T. F. Pajak, A. A. Forastiere, et al., "PostOperative Concurrent Radiotherapy and Chemotherapy in High Risk Squamous Cell Carcinoma of the Head and Neck," The New England Journal of Medicine, Vol. 350, No. 19, 2004, pp. 1937-1944. http://dx.doi.org/10.1056/NEJMoa032646

[2] J. Bernier and S. M. Benzten, "Post-Operative Treatment of Locally Advanced Head and Neck Cancers," In: D. J. Adelstien, Ed., Current Clinical Oncology: Squamous Cell Head and Neck Cancer, Humana Press Inc., Totwa, 2005, pp. 197-210.

http://dx.doi.org/10.1007/978-1-59259-938-7_14

[3] A. Lavaf, E. M. Genden, J. A. Cesaretti, et al., "Adjuvant Radiotherapy Improves Overall Survival for Patients with Lymph Node-Positive Head and Neck Squamous Cell Carcinoma," Cancer, Vol. 112, No. 3, 2008, pp. 535-543. http://dx.doi.org/10.1002/cncr.23206

[4] J. Kao, A. Lavaf, M. S. Teng, et al., "Adjuvant Radiotherapy and Survival for Patients with Node-Positive Head and Neck Cancer: An Analysis by Primary Site and Nodal Stage," International Journal of Radiation Oncology Biology Physics, Vol. 71, No. 2, 2008, pp. 362-370. http://dx.doi.org/10.1016/j.ijrobp.2007.09.058

[5] R. W. Hinerman, W. M. Mendenhall, C. G. Morris, et al., "Postoperative Irradiation for Squamous Cell Carcinoma of the Oral Cavity: 35-Year Experience," Head Neck, Vol. 26, No. 11, 2004, pp. 984-994. http://dx.doi.org/10.1002/hed.20091

[6] V. Sandro, M. B. B. S. Porceddu, F. R. A. N. Z. C. R. Belinda Campbell, M. B. B. S. Danny Rischin, M. B. B. S. F.R.A.C.P., et al., "Post-Operative Chemoradiotherapy for High Risk Head and Neck Squamous Cell," International Journal of Radiation Oncology Biology Physics, Vol. 60, No. 2, 2004, pp. 365-373.

http://dx.doi.org/10.1016/j.ijrobp.2004.03.011

[7] R. Fietkau, C. Lautenschlger, R. Sauer, J. Dunst, A. Becker, M. Baumann, T. Wendt, K. Grüschow, C. Hess, V. Budach, H. Iro, et al., "Postoperative Concurrent Chemotherapy versus Radiotherapy in High-Risk SCCA of the Head and Neck: Results of the German Phase III Trial ARO 96-3," Journal of Clinical Oncology, 2006 ASCO Annual Meeting Proceedings Part I, Vol. 24, No. 18S, 2006, p. 5507.

[8] M. D. Branko Zakotnik, M. D. Marjan Budihna, L. Smid, et al., "Patterns of Failures in Patients with Locally Advanced Head and Neck Cancer Treated Postoperatively with Irradiation or Concomitant Irradiation with Mitomycin D and Bleomycin," International Journal of Radiation Oncology Biology Physics, Vol. 67, No. 3, 2007, pp. 675-690. 